\title{
Distributed-Feedback Grating Used as an Array-Mode Selector in Resonant Antiguided Diode Laser Arrays: Effects of the Mirror Facet Position with Respect to the Grating
}

\author{
M. P. Nesnidal, L. J. Mawst, D. Botez, Fellow, IEEE, and J. Buus
}

\begin{abstract}
In resonant and nearly resonant antiguided arrays (ROW arrays), if the grating is located below the active region, a distributed-feedback (DFB) grating can function as a selector for the in-phase array mode in addition to its role as a frequency discriminator. Threshold current densities are calculated for competing (lateral) spatial modes in $\mathrm{AR} / \mathrm{HR}$ coated 10- and 20-element arrays incorporating lower gratings (ROW-LDFB arrays). Calculations include the effects of random facet locations relative to the grating phase. The threshold current density, $J_{\mathrm{th}}$, of the in-phase mode and its discrimination, $\Delta J_{\mathrm{th}}$, against other (array) spatial modes vary with facet location. For 20 (10) elements, $J_{\text {th }} \approx 350-525(400-550) \mathrm{A} / \mathrm{cm}^{2}$ and $\Delta J_{\text {th }}$ reaches a maximum of $55(58) \mathrm{A} / \mathrm{cm}^{2}$ for $0.01 / 0.95 \mathrm{AR} / \mathrm{HR}$-coated 350 $\mu \mathrm{m}$-long devices. For 10-element arrays, the probability of inphase operation is approximately $50 \%$; for 20 -element arrays, the probability is $100 \%$. Preliminary experimental results from 20-element nonresonant devices with uncoated facets are near diffraction-limited beams and nearly single-frequency operation to $250 \mathrm{~mW}$.
\end{abstract}

Index Terms-Distributed feedback lasers, phase-locked oscillators, semiconductor laser arrays, spatial filters.

$\mathbf{L}$ ARGE-APERTURE, resonant and nearly resonant antiguided diode laser arrays (so-called ROW arrays) have demonstrated the ability to operate in-phase with stable, diffraction-limited beams to record-high pulsed and continuous-wave (CW) output powers [1] due primarily to their high effective-index step $\left(\Delta n_{\text {eff }} \approx 0.05\right)$. The desired spatial-mode operation is achieved by designing the array such that the in-phase mode is laterally resonant. Discrimination against higher order spatial modes is usually provided by placing loss in the high effective-index interelement regions, suppressing modes with significant interelement field. We have previously demonstrated [2] the first simultaneous singlefrequency and single-spatial-mode operation from resonant antiguided arrays by employing distributed-feedback (DFB) for frequency discrimination and interelement loss for spatialmode selection (i.e., ROW-DFB arrays). It has been shown [3], however, that the presence of interelement loss could lead

Manuscript received September 10, 1997; revised December 11, 1997. This work was supported by the National Science Foundation under Grant ECS9522035.

M. P. Nesnidal, L. J. Mawst, and D. Botez are with the Reed Center for Photonics, University of Wisconsin-Madison, Madison, WI 53706 USA.

J. Buus is with Gayton Photonics Ltd., Gayton, Northants NN7 3EZ, U.K. Publisher Item Identifier S 1041-1135(98)02435-5.

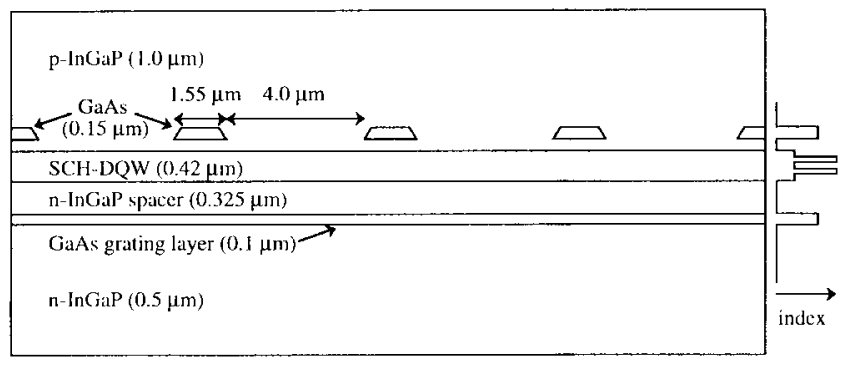

Fig. 1. Cross-sectional schematic of a ROW array with a lower DFB grating for use as an array-mode selector. The transverse element field has a greater overlap with the grating layer compared to that for the interelement field. This results in greater feedback for modes with more element field. The amount of coupling is adjusted by varying the $\mathrm{n}$-InGaP spacer thickness.

to self-pulsations due to saturable absorption at high drive levels. High-power coherent operation of such arrays could, therefore, benefit from novel spatial-mode selection that does not rely on absorbing interelement regions.

We have also reported [4] that a DFB grating located below the active region in ROW arrays (i.e., ROW-LDFB arrays) could provide not only frequency discrimination, but would also serve as a (lateral) spatial-mode selector, eliminating the need for interelement loss and other potential sources of self-pulsations [5]. The role of the lower DFB grating as a spatial-mode discriminator relies on the fact that the amount of optical field overlap with the lower grating is array-mode dependent [4]. The grating coupling coefficient is calculated for each array mode from the respective transverse fields in the element and interelement regions by using [4, eq. (1)], similar to [6, eq. (7.3.43)].

The previous analysis [4] neglected the effects of finite facet reflectivities and assumed that cavity lengths were integer multiples of the grating period, $L=n \Lambda$ (i.e., precise and identical cleave locations relative to the grating phase). The more realistic analysis presented here includes both the effects of AR/HR coatings as well as the statistical implications of random cleave locations relative to the grating phase. Furthermore, by comparison to prior work, the analysis is extended from 10- to 20-element arrays.

A schematic representation of the ROW-LDFB array structure analyzed here is shown in Fig. 1. The active region consists of a DQW-SCH structure incorporating 200-nm-thick 


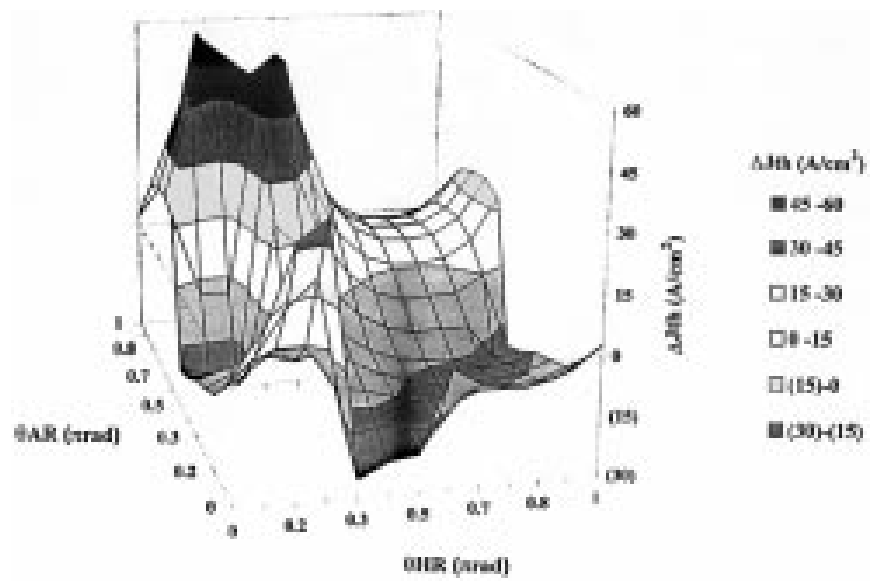

Fig. 2. Mode discrimination versus facet location for a 10-element ROW-LDFB with 0.01/0.95 AR/HR-coated facets. The resonant in-phase array mode operating in a single longitudinal DFB mode is favored approximately $50 \%$ of the time. Otherwise, the lower out-of-phase array mode (i.e., mode 9) operating in a single longitudinal DFB mode is favored.

InGaAsP optical confinement layers surrounding two 7-nmthick InGaAs quantum wells. The upper and lower cladding layers are 1000- and 500-nm-thick InGaP, respectively, and the substrate is GaAs. A second-order sinusoidal DFB grating, with a tooth height of $75 \mathrm{~nm}$, is defined above the lower cladding layer and planarized with a 100-nm-thick GaAs layer which is separated from the lower confinement layer by a 325-nm-thick InGaP spacer layer. The high effective-index interelement regions are defined by $150-\mathrm{nm}$-thick GaAs passive guide regions separated from the upper confinement layer by a $180-\mathrm{nm}$-thick InGaP spacer layer, yielding an effectiveindex step between regions of 0.026 . Element and interelement widths are 4.0 and $1.55 \mu \mathrm{m}$, respectively, corresponding to the in-phase mode resonance.

The mode behavior of ROW arrays was discussed previously [7]. Array modes are named according to the number of intensity nulls. For 10 (20)-element arrays, in addition to the desired in-phase array mode 18 (38), the competing spatial modes considered in this analysis are the upper and lower outof-phase modes 27 and 9 (57 and 19) and the upper and lower adjacent modes 19 and 17 (39 and 37).

For each array mode $\mathrm{m}$, the edge radiation loss, $\alpha_{\mathrm{rad}, m}$, the 2-D field intensity overlap with the quantum wells, $\Gamma_{2-\mathrm{D}, m}$, and the normalized coupling coefficient, $\kappa L_{m}$, are calculated [8] for both 10- and 20-element arrays. Calculation of an effective mirror loss $2 \gamma_{m}^{\prime}$ for each array mode is accomplished using the coupling coefficients [9]. For all cases, the device length is $350 \mu \mathrm{m}$. The threshold gain, $g_{\mathrm{th}, m}$, and threshold current density, $J_{\mathrm{th}, m}$, for mode $m$ are then given by

$$
\begin{aligned}
g_{\mathrm{th}, m} & =\frac{2 \gamma_{m}^{\prime}+\alpha_{\mathrm{rad}, m}}{\Gamma_{2-\mathrm{D}, m}} \\
J_{\mathrm{th}, m} & =J_{o}+\frac{g_{\mathrm{th}, m}}{B}
\end{aligned}
$$

where $J_{0} \approx 150 \mathrm{~A} / \mathrm{cm}^{2}$ is the transparency current density and $B \approx 4.5 \mathrm{~cm} / \mathrm{A}$ is the gain coefficient. The internal cavity loss as well as radiation loss due to first-order diffraction are assumed to be negligible compared to the other losses.

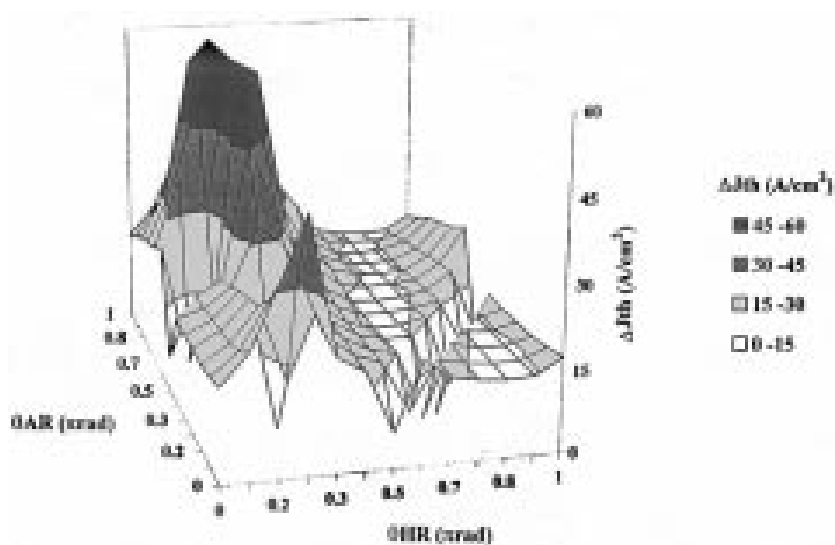

Fig. 3. Mode discrimination versus facet location for a 20-element ROW-LDFB with 0.01/0.95 AR/HR-coated facets. The resonant in-phase array mode dominates the entire parameter range. For nearly all instances, the in-phase array mode operates in a single longitudinal DFB mode. Degenerate longitudinal DFB modes occur for $\left(\theta_{\mathrm{AR}}, \theta_{\mathrm{HR}}\right)=(0.25 \pi, 0.25 \pi)$ and $(0.25 \pi, 0.75 \pi)$; i.e., single-frequency operation cannot occur at those phase angles.

Equations (1) and (2) describe the threshold condition for array modes experiencing optical feedback through DFB interaction. The structure is designed such that the in-phase array mode operating in a longitudinal DFB mode has the lowest threshold current density of all DFB modes. To insure DFB action, the in-phase DFB mode is also compared to all array modes operating in longitudinal Fabry-Perot modes by replacing the effective mirror loss in (1) with the modeindependent mirror loss $(2 L)^{-1} \ln \left(R_{1}^{-1} R_{2}^{-1}\right)$ at the facets.

Fig. 2 shows the mode discrimination for a 10-element ROW-LDFB array with 0.01/0.95 AR/HR coatings. The difference in threshold current density, $\Delta J_{\mathrm{th}}$, between the in-phase mode 18 and the next closest mode is plotted as a function of both facet locations, $\theta_{\mathrm{AR}}$ and $\theta_{\mathrm{HR}}$, relative to the grating phase. For each array mode and for all $\theta_{\mathrm{AR}}$ and $\theta_{\mathrm{HR}}$ values, several longitudinal DFB modes were considered in addition to the longitudinal Fabry-Perot modes. The special case of $\theta_{\mathrm{AR}}=\theta_{\mathrm{HR}}=0$ corresponds to the previous analysis [4]. The array operates in the in-phase mode 18 or the lower out-ofphase mode 9 as the facet locations vary independently within the unique range 0 through $\pi$ radians. Typical values of the threshold current density for the in-phase mode are 400-550 $\mathrm{A} / \mathrm{cm}^{2}$. The discrimination against the out-of-phase mode reaches a maximum value of $58 \mathrm{~A} / \mathrm{cm}^{2}$ near $\left(\theta_{\mathrm{AR}}, \theta_{\mathrm{HR}}\right)=$ $(0.75 \pi, 0.25 \pi)$. Since cleave location is inherently random, Fig. 2 provides yield information and shows that in-phase DFB mode operation is favored approximately $50 \%$ of the time in 10-element ROW-LDFB arrays incorporating 0.01/0.95 AR/HR coatings.

The competition between the in-phase and lower out-ofphase modes exhibited in Fig. 2 can be explained by (1) and the values of $\alpha_{\mathrm{rad}, m}$ for the two modes near the in-phase mode resonance. Although the in-phase mode experiences the most optical feedback (lowest $2 \gamma_{m}^{\prime}$ ) and has the highest overlap with the active region (highest $\Gamma_{2 \text {-D, } m}$ ), the edge radiation loss reaches its maximum at resonance [7]. The lower out-of-phase mode has higher values for $2 \gamma_{m}^{\prime}$, but $\alpha_{\mathrm{rad}, m}$ is virtually zero 


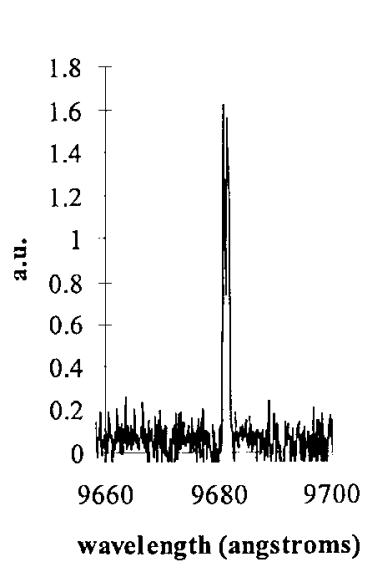

(a)

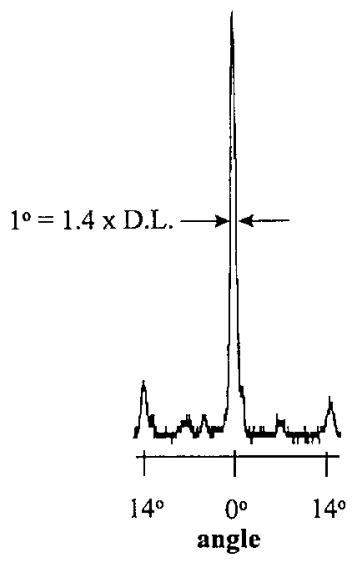

(b)
Fig. 4. Experimental data from a 20-element nonresonant LDFB array with uncoated facets. $250 \mathrm{~mW}$ was measured from both facets. The far-field profile is $1.4 \times \mathrm{D}$. L. and a spectral-line temperature coefficient of $0.6 \AA /{ }^{\circ} \mathrm{C}$ is measured, confirming DFB action. Nearly single-frequency operation is observed at $968 \mathrm{~nm}$. (a) Spectrum. (b) Far-field profile.

[7]. Since the edge radiation loss of the in-phase mode at its resonance is inversely proportional to the number of elements [7], (1) suggests that increasing the number of elements from 10 to 20 would significantly decrease its $J_{\text {th }, m}$ value and thus allow distributed feedback to become the dominant spatialmode discriminator.

The effect of increasing the number of elements is shown in Fig. 3, which plots the mode discrimination for 20-element ROW-LDFB arrays with 0.01/0.95 AR/HR coatings. In contrast to Fig. 2, in-phase mode operation is now favored for all cleave locations. Threshold current densities for in-phase operation vary between $350-525 \mathrm{~A} / \mathrm{cm}^{2}$ and $\Delta J_{\text {th }}$ reaches a maximum of $55 \mathrm{~A} / \mathrm{cm}^{2}$ as a result of the lower edgeradiation loss. Although the lower out-of-phase mode 19 is, in general, still the most competitive, nulls in $\Delta J_{\text {th }}$ occur at certain cleave locations corresponding to degenerate inphase mode operation in two longitudinal DFB modes. In Fig. 3, degenerate operation of in-phase mode 38 occurs for $\left(\theta_{\mathrm{AR}}, \theta_{\mathrm{HR}}\right)=(0.25 \pi, 0.25 \pi)$ and $(0.25 \pi, 0.75 \pi)$; i.e., singlefrequency operation cannot occur at those phase angles.

As the reflectivity of the AR coating increases, the threshold current densities and the overall discrimination against the lower out-of-phase mode decrease. Although not yet completely understood, this behavior can be partially explained by the fact that $J_{\text {th }}$ for the lower out-of-phase mode decreases more rapidly compared to the in-phase mode as the reflectivity of the AR coating increases. For a $3 \%$ AR coating, $J_{\text {th }}$ for the in-phase mode varies between $325-475 \mathrm{~A} / \mathrm{cm}^{2}$ and $\Delta J_{\mathrm{th}}$ reaches a maximum value of $21 \mathrm{~A} / \mathrm{cm}^{2}$. Even though the amount of intermodal discrimination is relatively small, single spatial-mode operation is assured to high powers due to the immunity of the resonant in-phase mode to multimoding via gain spatial hole burning [10]. For a 5\% AR coating, $J_{\text {th }}$ for the in-phase mode varies between $300-425 \mathrm{~A} / \mathrm{cm}^{2}$ and $\Delta J_{\text {th }}$ reaches a maximum value of $11 \mathrm{~A} / \mathrm{cm}^{2}$. In both cases, the in-phase mode is still favored for all cleave locations with degenerate operation occurring at certain locations. In addition to degeneracy at $\left(\theta_{\mathrm{AR}}, \theta_{\mathrm{HR}}\right)=(0.25 \pi, 0.25 \pi)$ and $(0.25 \pi, 0.75 \pi)$ as in Fig. 3, an additional degeneracy at $\left(\theta_{\mathrm{AR}}, \theta_{\mathrm{HR}}\right)=(0.75 \pi, 0.25 \pi)$ exists for both $3 \%$ and $5 \% \mathrm{AR}$ coatings.

Preliminary data are shown in Fig. 4 for a 20-element LDFB array with uncoated facets. Element and interelement widths are 3 and $1 \mu \mathrm{m}$, respectively, and the device length is $500 \mu \mathrm{m}$. The measured farfield profile is $1.4 \times$ diffraction limit (D.L.) $\left(1^{\circ}\right.$ versus $0.7^{\circ}$ for D.L. lobewidth), indicating nonresonant array operation [11]. The measured output power was $250 \mathrm{~mW}$ from both facets with $80 \%$ of the emission in the main lobe. This behavior is similar to nonresonant arrays [11] except, due to the DFB grating, there is no need for a Talbottype filter for suppressing the out-of-phase mode. There is simultaneous oscillation of the in-phase and upper adjacent modes, as confirmed by the two-mode frequency spectrum occurring at $968 \mathrm{~nm}$. A spectral-line temperature coefficient of $0.6 \AA /{ }^{\circ} \mathrm{C}$ is measured, confirming DFB action.

The effects of real facets on mode discrimination in 10- and 20-element ROW-LDFB arrays have been analyzed. For 10 (20)-element arrays, in-phase mode operation occurs approximately $50 \%(100 \%)$ of the time for random cleave locations in $0.01 / 0.95 \mathrm{AR} / \mathrm{HR}$ coated devices. $J_{\mathrm{th}}$ of the in-phase mode ranges between $400-550(350-525) \mathrm{A} / \mathrm{cm}^{2}$ and $\Delta J_{\mathrm{th}}$ reaches a maximum of $58(55) \mathrm{A} / \mathrm{cm}^{2}$.

\section{REFERENCES}

[1] D. Botez, "Monolithic phase-locked semiconductor laser arrays," in Diode Laser Arrays, D. Botez and D. R. Scifres, Eds. Cambridge, U.K.: Cambridge Univ. Press, 1994, ch. 1, pp. 1-71.

[2] M. P. Nesnidal, L. J. Mawst, A. Bhattacharya, D. Botez, L. DiMarco, J. C. Connolly, and J. H. Abeles, "Single-frequency, single-spatial-mode ROW-DFB diode laser arrays," IEEE Photon. Technol. Lett., vol. 8, pp. 182-184, 1996

[3] S. Ramanujan, H. G. Winful, M. Felsky, R. K. DeFreez, D. Botez, M. Jansen, and P. Wisseman, "Temporal behavior of resonant-opticalwaveguide phase-locked diode laser arrays," Appl. Phys. Lett., vol. 64, pp. 827-829, 1994.

[4] M. P. Nesnidal, L. J. Mawst, and D. Botez, "Distributed-feedback grating used as a lateral-mode selector in phase-locked antiguided arrays," IEEE Photon. Technol. Lett., vol. 9, pp. 34-36, 1997.

[5] R. F. Nabiev, M. D. Iturbe-Castillo, J. J. Sanchez-Mondragon, A. I. Onishchenko, and D. Botez, "Nonlinear restrictions of Talbot-like spatial filters in semiconductor laser arrays," Appl. Opt., vol. 32, pp. 4480-4484, 1993.

[6] G. P. Agrawal and N. K. Dutta, Semiconductor Lasers, 2nd Ed. New York: Van Nostrand Reinhold, 1993.

[7] D. Botez, L. J. Mawst, G. L. Peterson, and T. J. Roth, "Phaselocked arrays of antiguides: Modal content and discrimination," IEEE J. Quantum Electron., vol. 26, pp. 482-495, 1990.

[8] W. Streifer, D. R. Scifres, and R. D. Burnham, "Coupling coefficients for distributed feedback single- and double-heterostructure diode lasers," IEEE J. Quantum Electron., vol. 11, pp. 867-873, 1975.

[9] J. Buus, Single Frequency Semiconductor Lasers, Tutorial Texts in Optical Engineering. New York: SPIE Optical Engineering Press, 1990, vol. TT5, pp. 27-35.

[10] R. F. Nabiev and D. Botez, "Comprehensive above-threshold analysis of antiguided diode laser arrays," IEEE J. Select. Topics Quantum Electron., vol. 1, pp. 138-149, 1995.

[11] L. J. Mawst, D. Botez, M. Jansen, T. J. Roth, and G. Peterson, "Highpower, narrow single-lobe operation from 20-element phase-locked arrays of antiguides," Appl. Phys. Lett., vol. 55, pp. 2060-2062, 1989. 